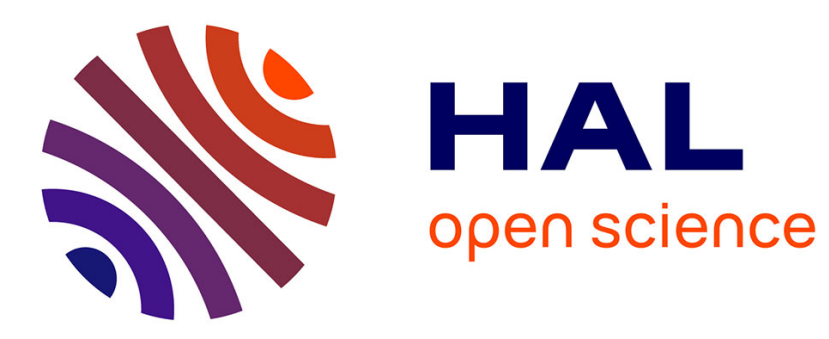

\title{
Fluctuations of the mass of the infinite cluster at percolation
}

\author{
A. Kapitulnik, N. Frid, G. Deutscher
}

\section{To cite this version:}

A. Kapitulnik, N. Frid, G. Deutscher. Fluctuations of the mass of the infinite cluster at percolation. Journal de Physique Lettres, 1984, 45 (9), pp.401-407. 10.1051/jphyslet:01984004509040100 . jpa00232361

\section{HAL Id: jpa-00232361 https://hal.science/jpa-00232361}

Submitted on 1 Jan 1984

HAL is a multi-disciplinary open access archive for the deposit and dissemination of scientific research documents, whether they are published or not. The documents may come from teaching and research institutions in France or abroad, or from public or private research centers.
L'archive ouverte pluridisciplinaire HAL, est destinée au dépôt et à la diffusion de documents scientifiques de niveau recherche, publiés ou non, émanant des établissements d'enseignement et de recherche français ou étrangers, des laboratoires publics ou privés. 


\title{
LE JOURNAL DE PHYSIQUE-LETTRES
}

J. Physique Lett. 45 (1984) L-401 - L-407

ler MAI 1984, PAGE L-401

Classification

Physics Abstracts

$05.50-71.50-73.90$

\section{Fluctuations of the mass of the infinite cluster at percolation}

\author{
A. Kapitulnik $\left(^{*}\right)$, N. Frid and G. Deutscher \\ Department of Physics and Astronomy, Tel-Aviv University, Ramat-Aviv, \\ Tel-Aviv 69978, Israel
}

(Reçu le 28 décembre 1983 accepté le ler mars 1984)

\begin{abstract}
Résumé. - Nous avons mesuré les fluctuations de la masse $M$ d'un amas infini de percolation, ainsi que celles d'objets fractals et de couches minces discontinues de $\mathrm{Pb}$. On trouve que ces fluctuations divergent en fonction de l'échelle de longueur avec un exposant égal au double de la dimension fractale. Ce résultat est expliqué par un argument de loi d'échelle.
\end{abstract}

\begin{abstract}
We have measured the fluctuations of the mass $M$ of the infinite cluster in percolation, as well as those of fractal objects and discontinuous thin films of $\mathrm{Pb}$. It was found that these fluctuations diverge with the length scale with an exponent equal to twice the fractal dimensionality. A scaling argument is given to explain that result.
\end{abstract}

Geometrical fluctuations in the context of percolation were discussed previously by Coniglio, et al. [7] when they studied the fluctuations in the cluster numbers. Stauffer [2] and then Coniglio and Stauffer [3] analysed further data and presented results on the investigation of the mean cluster size which is the analogous of the susceptibility in an ordinary phase transition. They related that data to the fluctuations in the mass of percolating clusters.

In this paper we use another approach which is based on the investigation of correlations within a fractal object. By relating these correlations to the overall two point correlation function we can show the existence of the fluctuations dissipation theorem for that problem. We further show the relation between that result and the former results on the mean cluster size. We conclude that

$$
\overline{\Delta M^{2}} \equiv \overline{(M(L)-\overline{M(L)})^{2}} \propto L^{2 D}
$$

$\left({ }^{*}\right)$ Permanent address : Institute for Polymers and Organic Solids and Institute for Theoretical Physics, University of California, Santa Barbara, California 93106, U.S.A. 
where $M(L)$ is the mass within a volume $L^{d}$ (with linear size $L$ ) that surrounds an occupied point of the fractal object, having a fractal dimensionality $D$ [4-7].

We shall demonstrate the validity of equation (1) by using scaling arguments as well as direct measurements on three objects : a) the Sierpinski gasket [7]; b) the infinite cluster in percolation ; and c) a discontinuous thin film of $\mathrm{Pb}$ deposited on amorphous $\mathrm{Ge}$.

To start, let us first derive equation (1). To do so, we will concentrate on the percolation problem.

We define by $\rho(r)$, the conditional probability that if a point at the origin is occupied by the infinite cluster, a point at a distance $r$ from it also belongs to that cluster. It was shown by Kapitulnik et al. [7] that for length scales $r$, small compared to the correlation length $\xi$ but still large compared to the lattice spacing, we expect

$$
\rho(r) \propto r^{-\beta / v}
$$

where $\beta$ is the infinite cluster density exponent $\left(P_{\infty} \propto\left(p-p_{\mathrm{c}}\right)^{\beta}\right)$ and $v$ is the correlation length exponent $\left(\xi \propto\left(p-p_{c}\right)^{-v}\right)$. The mass. $M(L)$, defined above is found via a straightforward integration [7] $M(L) \propto \int_{0}^{L} \rho(r) \mathrm{d}^{d} r \sim L^{d-\beta / v}=L^{D}$. It was then concluded that :

$$
\bar{\rho}(L) \equiv \frac{\overline{M(L)}}{L^{d}} \propto \rho(r=L)
$$

in particular, this is true for $L>\xi$ where $\rho(L) \propto P_{x} \propto Q$ where $Q$ is the order parameter, e.g., as defined by the $q \rightarrow 1$ limit of the $q$ states Potts model [11]. We thus suggest that $\rho(r)$ has the finite size form of the order parameter in percolation [11]. This fact is highly plausible because at length scales below $\xi$, for any cluster, as long as we do not reach its boundaries we observe the same distribution of mass as for the infinite clusters. (The above suggestion can be immediately generalized to any other $q$ states Potts model like of the Ising model [11]). To be more specific, the relation between $\rho(r)$ and the overall two points correlation function, $G(r)$, was shown by Aharony, Gefen, and Kapitulnik to be (apart from exponentially decaying factors)

$$
\rho(r) \cdot\left(\sum_{s=s_{r}} s n_{s}+P_{\infty}\right)=G(r)+P_{\infty}^{2}
$$

where $s_{r} \propto r^{D}$ is a cutoff cluster size. Certainly this functional dependence is defined also below the percolation threshold $\left(P_{\alpha}=0\right)$. For $r \rightarrow \infty$ it gives $P_{\infty}$ above $P_{\mathrm{c}}$.

The above arguments suggest a simple way to calculate the fluctuations of the mass since these fluctuations arise from the fluctuations of the order parameter.

By the use of the fluctuation-dissipation theorem [8] we write that the amount of fluctuation of $Q$ per unit volume $\xi^{d}$ should be proportional to the response function, namely to the susceptibility $\chi$.

$$
\xi^{d} \cdot\left[\overline{Q^{2}}-\bar{Q}^{2}\right] \propto \chi \propto \xi^{\gamma / v} .
$$

To calculate now the fluctuations in the function $\rho(r)$, the straightforward way is to take the finite size version of the equation (5), namely replace $\xi$ by $L$ and $Q$ by $\rho(L)$.

Thus :

$$
L^{d} \cdot\left[\overline{\rho^{2}(L)}-\overline{\rho(L)}^{2}\right] \propto \chi(L) \propto L^{\gamma / v} .
$$

Using now the hyperscaling relation $\gamma=d v-2 \beta$ and the definition (3), we find

$$
\overline{\Delta M^{2}} \propto L^{2 d-2 \beta / v}
$$

from which equation (1) immediately follows. 
With only a little of algebra one can arrive at equation (6) by using equation (4) and integrating directly both $\rho(r)$ and $\rho^{2}(r)$ and averaging. By doing so, one can immediately see the role $\rho(r)$ plays and its connection to the susceptibility both above and below $P_{\mathrm{c}}$.

The above treatment allows us therefore to generalize the applicability of the fluctuationdissipation theorem to all concentration regimes. In particular, any fractal we can treat as being at criticality and thus attribute to it an equation similar to equation (6).

We must emphasize that the above arguments might be very different for $d \geqslant 6$ as hyperscaling breaks down [11]. It is straightforward to show that for $d \geqslant 6$

$$
\overline{\Delta M^{2}} \propto L^{8} .
$$

Again, since $D=4$ for $d>6$ [11] we recover equation (1). In the form (1), one can generalize the expression for $\overline{\Delta M^{2}}$ to any fractal object since only the fractal dimensionality enters that expression.

In what follows, we shall present Monte Carlo simulations on a $2 D$ percolation cluster as well as measurements on a regular fractal to confirm equation (1).

The general method we have used was to take one fractal object filling a $512 \times 512$ matrix and studying it, using the method suggested above. A square of size $128 \times 128$ was moved randomly on the object. Whenever its centre coincided with the object, a counting process of the number of occupied points $M(L)$, in a square of size $L(3<L<128)$ was performed. We then averaged $M(L)^{2}$ and calculated $\overline{\Delta M^{2}}=\overline{(M(L)-\overline{M(L)})^{2}}$.

\section{The Sierpinski gasket.}

The Sierpinski gasket (Fig. 1) was studied previously in its random version by Kirkpatrick [5] as a model for the backbone in percolation. It was also pointed out by Kapitulnik et al. [9] that

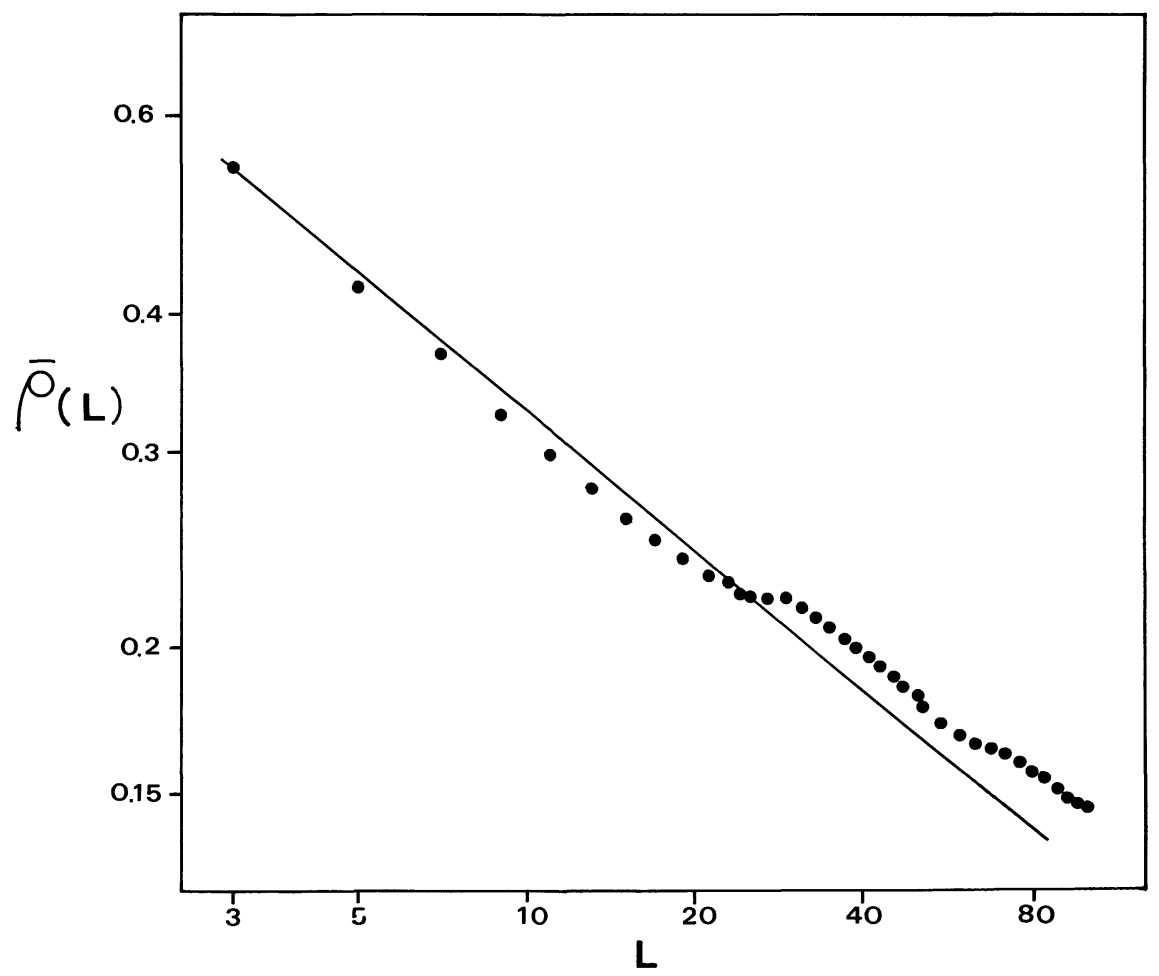

Fig. 1. - Density $\bar{\rho}(L)$ versus $L$ for the random Sierpinski gasket $(D=\log 3 / \log 2)$. 
when performing the calculation of $\bar{\rho}(L)$ on that object, an oscillatory behaviour of the mass with the length scale is observed. Here, we have generated a $512 \times 512,2 D$ matrix, built of 16 squares, each of seven iterations of the gasket. We have investigated both, the regular and the random version. Figure 3, in reference [9], shows the measurement of $\bar{\rho}(L)$ on the regular fractal. One clearly observes these "jumps" in $\bar{\rho}(L)$ at the sizes of the "holes " $L=8,16,32,64,128$. We expect such oscillations to appear whenever the fractal is regular, exhibiting certain sizes of holes. In contrast with that figure, we thus show figure 1 , where $\bar{\rho}(L)$ is presented for the random version of the same gasket. Clearly the oscillations have disappeared. In figure 2, we present the measurement of $\overline{\Delta M^{2}}$ and indeed one finds the slope to be $3.20 \pm 0.05$ in agreement with equation (1) (note that for this fractal $D=\log 3 / \log 2 \cong 1.58$ ).

\section{The infinite cluster in percolation.}

We have used two methods to investigate the validity of equation (1) for the infinite cluster in percolation. The first was initially used by Kapitulnik et al. [9] for the study of the crossover from self similarity to homogeneity in the infinite cluster.

We studied two dimensional $(d=2)$ Monte Carlo simulations of site percolation on a triangular lattice, with $p>p_{\mathrm{c}}=0.5$. For each desired value of $p$ we have generated 30000 samples of size $201 \times 201$, and kept only those (typically more than 2000 ) whose actual concentration was within $0.05 \%$ of $p$. (Without this selection, the variation is of order $0.5 \%$, and the crossover effects are smeared. See e.g. Ref. [9]). We next identified the largest cluster on each of the remaining samples, picked only those samples in which the central site belonged to this cluster, and counted the sites connected to it within squares of size $L$ around it, $M(L)$. Averaging $M(L)$ and $M^{2}(L)$ over

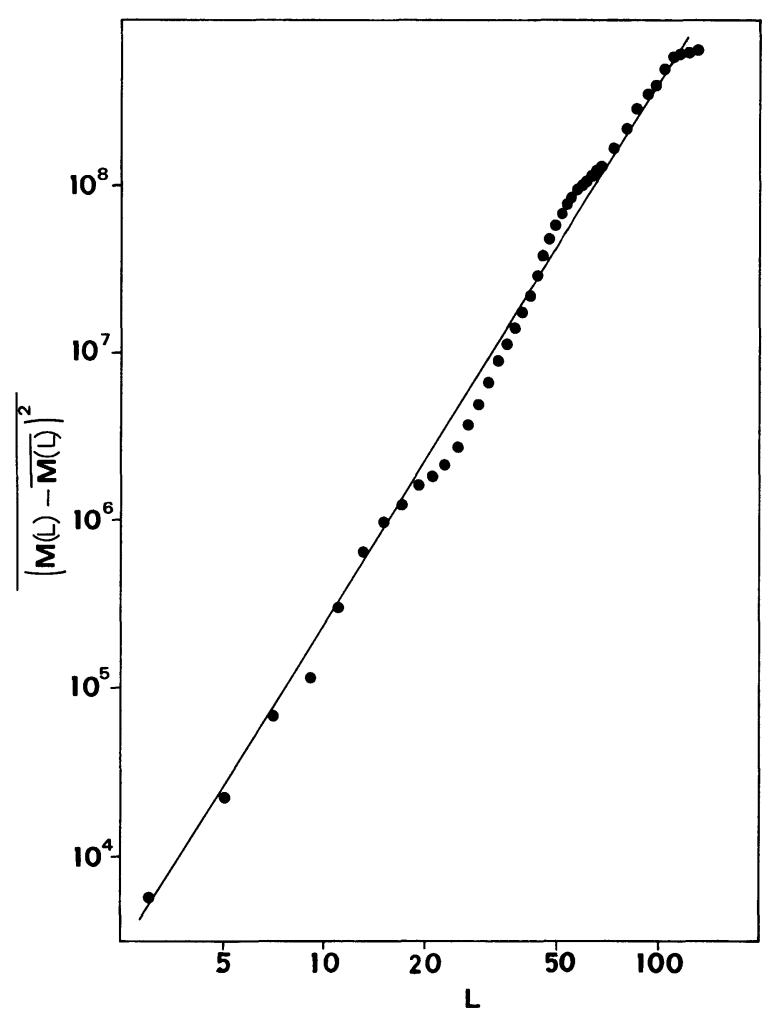

Fig. 2. - Mass fluctuations, $\overline{\Delta M^{2}}$ in arbitrary units versus $L$ for the gasket in Fig. 1. 
these samples (whose number was of order 1000), we found the average density $\bar{\rho}(L)$ and the average of the square of the density $\overline{\rho^{2}}(L)$. Figure 3 shows typical plots of $\bar{\rho}(L)$ and $\overline{\Delta \rho^{2}} \equiv(\rho(L)-$ $\bar{\rho}(L))^{2}$ for $p-p_{\mathrm{c}}=0.005$. Similar plots were found for other concentrations in the self similar region $(L<\xi)$. We have found $\bar{\rho}(L) \propto L^{-0.10 \pm 0.015}$ and $\overline{\Delta \rho^{2}} \propto L^{-0.20 \pm 0.015}$. Both results are in excellent agreement with predicted values for $D$ [1-7] and hence confirm equation (1).

The second method of investigation of the infinite cluster is similar to that used for the gasket. We produced $512 \times 512$ site percolation samples on a square lattice at different concentrations $p>p_{\mathrm{c}}=0.5927$. We next identified the largest cluster on each sample. Then as explained before we calculate $\overline{\Delta M(L)^{2}}$. A typical result for $p-p_{\mathrm{c}}=0.015$ is shown in figure 4 . The resulting exponent was found to be $3.80 \pm 0.02$ again in good agreement with equation (1). The deviation for large $L$ is due to the crossover to homogeneity at $\xi$, the correlation length.

\section{Discontinuous film of $\mathrm{Pb}$ on amorphous Ge.}

The last object to be reported is the infinite cluster of a sample of $\mathrm{Pb}$ deposited on amorphous Ge above the continuity threshold.

For that investigation the samples were digitized by the use of a photodensitometer in line with a PDP/11 computer. A detailed description of the digitization technique as well as the experimental set up is given in references [12,8].

The scaling properties of discontinuous thin films of $\mathrm{Pb}$ were first investigated by Kapitulnik and Deutscher [8]. They established that percolation is relevant to the description of discontinuous metal films. The fractal dimension of $\mathrm{Pb}$ thin films was measured and it was found that $D=1.90$.

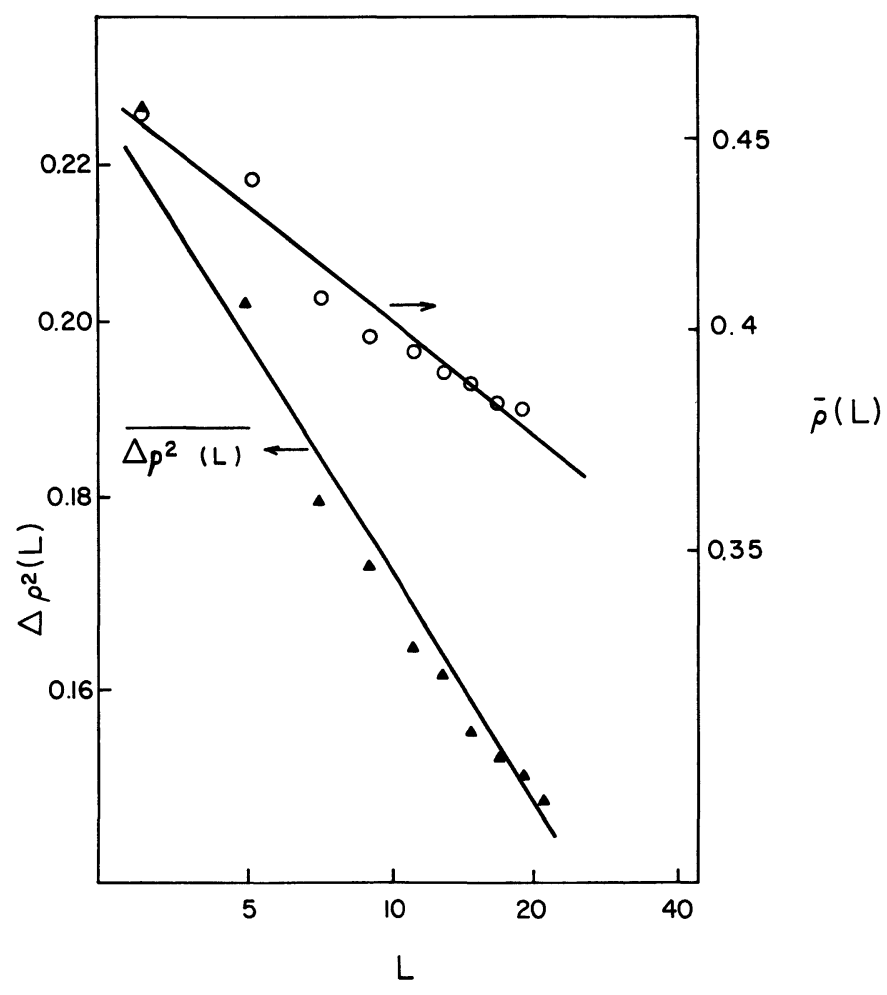

Fig. 3. - Density $\bar{\rho}(L)$ and density fluctuations $\overline{\Delta \rho^{2}}$ versus $L$ for the percolation infinite cluster (triangular lattice, $\left.p-p_{\mathrm{c}}=0.005\right)$. 


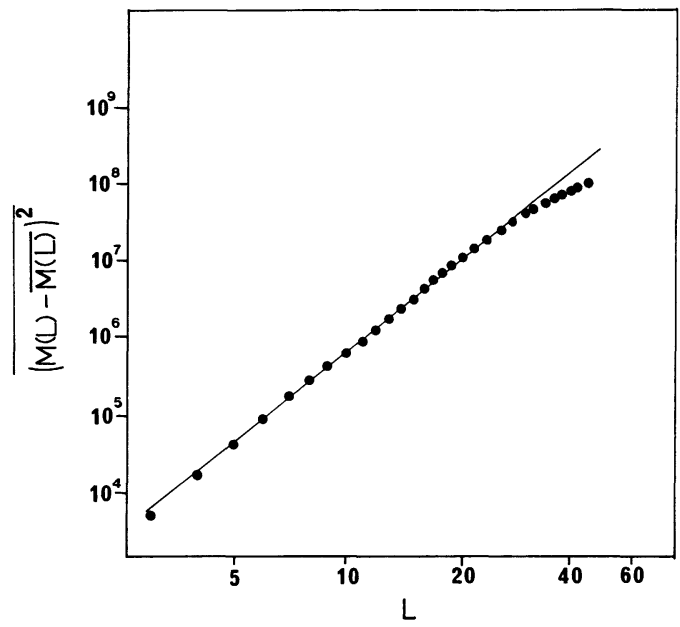

Fig. 4. - Mass fluctuations, $\overline{\Delta M^{2}}$, in arbitrary units for the percolating infinite cluster (square lattice, $\left.p-p_{\mathrm{c}}=0.015\right)$.

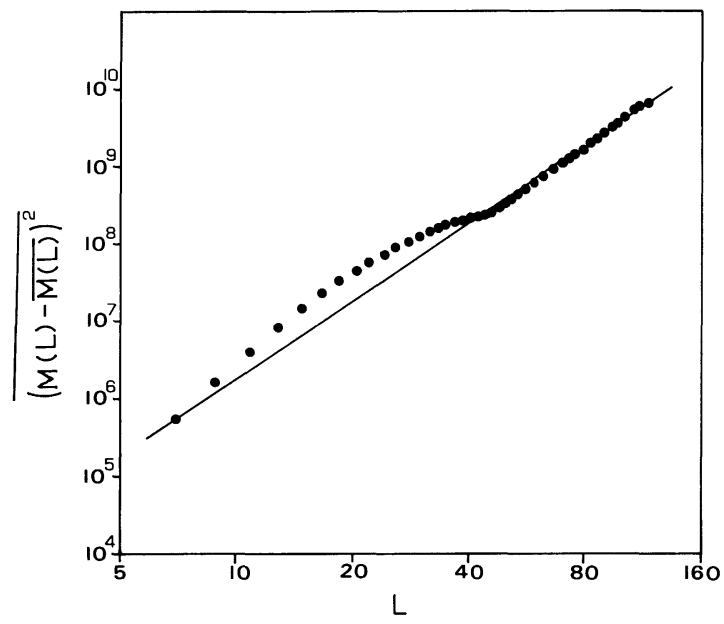

Fig. 5. - Mass fluctuations, $\overline{\Delta M^{2}}$, in arbitrary units for the infinite cluster of a sample of $\mathrm{Pb}$ deposited on amorphous Ge close (and above) the continuity threshold.

Figure 5 depicts the results for $\overline{\Delta M^{2}}$ as were measured for a typical discontinuous film of $\mathrm{Pb}$. We clearly see a power law behaviour with the value $3.8 \pm 0.1$. Although the result is not as accurate as that of $D$ it is consistent with (1).

For that sample $D$ was found to be $1.90 \pm 0.02$. We note that for small $L$ the deviation is large, while the resultant exponent is actually the straight line for large $L$.

The fact that for each surface coverage we use only one micrograph is a source of large deviations, since we cannot always expect the equilibrium distribution of $\mathrm{Pb}$ islands.

In conclusion we have constructed a scaling theory for the behaviour of the fluctuations of the mass of fractal objects. The agreement with (1) for all the cases that we have investigated is excellent. 


\section{Acknowledgments.}

We are indebted to A. Aharony and D. Stauffer for useful discussions.

\section{References}

[1] Coniglio, A., Stanley, H. E. and Stauffer, D., J. Phys. A 12 (1979) L-323.

[2] Stauffer, D., Z. Phys. B 37 (1980) 89.

[3] Stauffer, D., Phys. Rep. 51 (1979) 1.

[4] Coniglio, A. and Stauffer, D., Lett. Nuovo Cimento 28 (1980) 33.

[5] KirkPatrick, S., in Les Houches Summer School on Ill-Condensed Matter, ed. by R. Balian, R. Maynard and G. Toulouse (North-Holland, Amsterdam) 1979.

[6] Stanley, H., J. Phys. $A 10$ (1977) L-211.

[7] Stanley, H. E. and Coniglio, A., in Percolation Structures and Processes, eds. G. Deutscher, R. Zallen and J. Adler (ann. of the Israel Physical Society, Vol. 5983) p. 81.

[8] Kapitulnik, A. and Deutscher, G., Phys. Rev. Lett. 49 (1982) 1444.

[9] Kapitulnik, A., Aharony, A., Deutscher, G. and Stauffer, D., J. Phys. A 16 (1983) L-269.

[10] See e.g. MA, S. K., Modern Theories of Critical Phenomena (Benjamin) 1976, p. 457.

[11] Aharony, A., Gefen, Y. and Kapitulnik, A., "Scaling at percolation above 6 dimensions ", to appear in J. Phys. A.

See also Aharony, A., Phys. Rev. B 22 (1980) 400.

[12] Kapitulnik, A. and Deutscher, G., «Comments on Computer Investigation of Micrographs of Discontinuous Metal Films Near Their Continuity Threshold », to appear in Thin Solid Films. 